\title{
artigo
}

\section{Avaliação da força muscular respiratória e função pulmonar de pacientes com doença renal crônica submetidos à hemodiălise}

\author{
Evaluation of respiratory muscle strength and pulmonary function of patients with chronic kidney disease submitted \\ to hemodialysis
}

Evaluación de la fuerza muscular respiratoria y la función pulmonar de pacientes con enfermedad renal crónica sometidos a hemodiálises

\begin{abstract}
RESUMO
Objetivo: Avaliar a força muscular respiratória (FMR) e a função pulmonar (FP) de pacientes com doença renal crônica (DRC) submetidos à hemodiálise (HD). Métodos: Estudo transversal com 41 participantes. Foram verificadas as pressões inspiratória e expiratória máxima (PImáx; PEmáx), capacidade Vital Forçada (CVF) e volume expiratório forçado no primeiro segundo (VEF1). Resultados: Não há diferença significante entre homens e mulheres em relação ao percentual do previsto alcançado para PImáx, porém há em relação a PEmáx $(p=0,082 ; p=0,003)$. Quanto a FP constatou-se que há diferença significativa entre homens e muIheres, para ambas as variáveis CVF e VEF1 ( $p=0,034$ e p=0,024). Conclusão: Em relação à FMR, os pacientes com doença renal crônica em hemodiálise possuem fraqueza muscular respiratória, e quando comparados por gênero, os homens apresentaram força muscular inspiratória reduzida em relação ao previsto. Quanto a FP, as mulheres possuem valores abaixo dos padrões de normalidade comparada aos homens.
\end{abstract}

DESCRITORES: Doença Renal Crônica; Hemodiálise; Força Muscular Respiratória; Função Pulmonar.

\begin{abstract}
Objective: To assess respiratory muscle strength (RMS) and lung function (PF) in patients with chronic kidney disease (CKD) undergoing hemodialysis (HD). Methods: Cross-sectional study with 41 participants. Maximum inspiratory and expiratory pressures (MIP; MEP), Forced Vital Capacity (FVC) and forced expiratory volume in the first second (FEV1) were checked. Results: There is no significant difference between men and women in relation to the percentage of the predicted achieved for MIP, but there is in relation to $\operatorname{MEP}(p=0.082 ; p=0.003)$. As for $P F$, it was found that there is a significant difference between men and women, for both variables FVC and FEV1 $(p=0.034$ and $p=0.024)$. Conclusion: Regarding RMS, patients with chronic kidney disease on hemodialysis have respiratory muscle weakness, and when compared by gender, men had reduced inspiratory muscle strength compared to predict. As for PF, women have values below normal standards compared to men.
\end{abstract}

DESCRIPTORS: Chronic Kidney Disease; Hemodialysis; Respiratory Muscle Strength; Lung function.

\section{RESUMEN}

Objetivo: evaluar la fuerza de los músculos respiratorios (FMR) y la función pulmonar (FP) en pacientes con enfermedad renal crónica (ERC) sometidos a hemodiálisis (HD). Métodos: estudio transversal con 41 participantes. Se verificaron las presiones inspiratoria y espiratoria máxima (MIP; MEP), la Capacidad Vital Forzada (FVC) y el volumen espiratorio forzado en el primer segundo (FEV1). Resultados: No hay diferencia significativa entre hombres y mujeres en relación al porcentaje de lo esperado alcanzado para MIP, pero sí en relación a MEP ( $p=0.082 ; p=0.003)$. En cuanto al PF, se encontró que existe una diferencia significativa entre hombres y mujeres, para ambas variables FVC y FEV1 $(p=0.034$ yp $=0.024)$. Conclusión: Con respecto a la FMR, los pacientes con enfermedad renal crónica en hemodiálisis tienen debilidad de los músculos respiratorios y, en comparación por sexo, los hombres tenían una fuerza de los músculos inspiratorios reducida en comparación con lo previsto. En cuanto a la FP, las mujeres tienen valores por debajo de los estándares normales en comparación con los hombres.

DESCRIPTORES: Enfermedad Renal Crónica; Hemodiálisis; Fuerza de los músculos respiratorios; Función pulmonar.

RECEBIDO EM: 05/01/2021 APROVADO EM: 19/01/2021 


\section{Nataly Gurgel Campos}

Fisioterapeuta. Doutora em Ciências Médicas- UFC. Docente do Curso de Fisioterapia. Departamento de Fisioterapia-Universidade Federal do Ceará- UFC. Grupo de Pesquisa InspiraFisio-UFC. Master Program in Physiotherapy and Functioning, Federal University of Ceara, Fortaleza, Brasil.

ORCID: 0000-0002-6551-1165

\section{Lara Costa Leite}

Fisioterapeuta. Discente do Programa de pós-graduação em Fisioterapia e Funcionalidade da Universidade Federal do CearáUFC. Grupo de Pesquisa InspiraFisio-UFC.

ORCID:0000-0001-8983-4746.

\section{Ítalo Caldas Silva}

Fisioterapeuta. Mestre em Ciências Médicas- UFC. Grupo de Pesquisa InspiraFisio-UFC. ORCID: 0000-0001-7610-2124

\section{Rayana Fialho da Costa}

Fisioterapeuta. Discente do Programa de pós-graduação em Ciências Médicas da Universidade Federal do Ceará- UFC. Grupo de Pesquisa InspiraFisio-UFC.

ORCID:0000-0003-1785-4996

\section{Ellys Rhaiara Nunes Rebouças}

Fisioterapeuta. Residência multidisciplinar em Infectologia - ESP/CE. Grupo de Pesquisa InspiraFisio-UFC. ORCID:0000-0002-3730-3369

\section{Larissa Rocha Miranda}

Fisioterapeuta. Residência multidisciplinar em Infectologia - ESP/CE. Grupo de Pesquisa InspiraFisio-UFC. ORCID: 0000-0001-7081-9909

\section{Francisco Lazaro Arruda}

Fisioterapeuta. Discente do Programa de pós-graduação em Saúde Pública da Universidade Federal do Ceará- UFC. ORCID:0000-0003-3277-0782

\section{Paulo Romão Ribeiro da Silva}

Enfermeiro. Mestre em Gestão em Saúde pela Universidade Estadual do Ceará- UECE.

ORCID:0000-0001-6311-4070

\section{INTRODUÇÃO}

A doença renal crônica (DRC) é definida na diretriz de prática clínica da Kidney Disease Improving Global Outcomes (KDIGO) como anormalidades da estrutura ou função renal presentes por tempo superior a três meses e que causam sérias implicações para a saúde ${ }^{(1)}$.

$\mathrm{O}$ número de pacientes com doença renal crônica que precisaram de diálise cresceu de 42 mil, em 2000, para 122 mil no ano de 2016. No ano passado, 5,7 mil pessoas fizeram transplante de rim no país, quantidade que vem aumentando, em média, $10 \%$ de um ano para o outro, de acordo com a Sociedade Brasileira de Nefrologia (SBN).

É uma doença crônica que possui cará- ter progressivo e irreversível que provoca a perda da função renal. Essa última ocorre de maneira gradual, o que leva o indivíduo a ficar assintomático e/ou acarreta sintomas leves que podem passar despercebidos durante muito tempo ${ }^{(2,3)}$.

A DRC é classificada em diferentes estágios que levam em consideração a causa, a diminuição da taxa de filtração glomerular e a albuminúria. Além disso, a presença de um ou mais marcadores de lesão renal - anormalidades dos sedimentos de urina, eletrólitos e outras anormalidades devido a distúrbios tubulares, anormalidades detectadas por histologia, anormalidades estruturais detectadas por imagem e história de transplante de rim - também são usados para auxiliar no diagnóstico e classificação da doença ${ }^{(1)}$.
Os pacientes aompanhados com DRC no estadiamento VI para V devem ser orientados para o tratamento dialítico, na tentativa de evitar a ocorrência de complicações graves que podem levar à morte. A hemodiálise (HD) é o tratamento mais utilizado, que deve ser realizada pelos portadores de DRC por toda a vida ou até se submeterem a um transplante renal bem-sucedido. Apesar de promoverem a manutenção e o prolongamento da vida, as terapias renais substitutivas não oferecem uma cura para a doença e, a longo prazo, acabam por prejudicar a vida cotidiana e a qualidade de vida $(\mathrm{QV})$ do paciente ${ }^{(4)}$.

Indivíduos com DRC, em geral, desenvolvem a síndrome urêmica que afeta diversos sistemas corporais, inclusive o sistema respiratório. Estudos tem demons- 
trado que a DRC está associada a várias doenças respiratórias, tais como: edema pulmonar, derrame pleural, fibrose, calcificação do parênquima pulmonar e síndrome da apnéia do sono. Além disso, a HD também pode impactar negativamente na força muscular respiratória e função pulmonar do indivíduo ${ }^{(5,6)}$.

Em decorrência dessas e de outras alterações, esses pacientes têm redução da força e função muscular respiratória e periférica, bem como, redução no condicionamento cardiorrespiratório. Tais complicações limitam a participação em atividades de vida diária, consequentemente reduzindo a $\mathrm{QV}$, além de aumentar a mortalidade nos portadores da doença ${ }^{(7)}$.

Este estudo teve como objetivo avaliar a força muscular respiratória e a função pulmonar de pacientes com doença renal crônica em hemodiálise.

\section{MÉTODOS}

Estudo transversal com abordagem quantitativa conduzido em uma clínica de hemodiálise na cidade de Fortaleza- Ceará, região nordeste do Brazil. Foram incluídos participantes com diagnóstico clínico de Doença Renal Crônica, cadastrados no Setor de Hemodiálise da referida clínica, com idade > 18 e <60 anos; ambos os gêneros; que estavam em tratamento dialitico mais de três (3) meses; com capacidade cognitiva e física suficiente para executar os procedimentos avaliativos, não possuir diagnóstico de doença pulmonar obstrutiva crônica e aceitar participar da pesquisa após ter lido, compreendido e assinado o Termo de Consentimento Livre e Esclarecido- TCLE.

Como critérios de exclusão considerou-se aqueles indivíduos com história de infarto agudo do miocárdio há menos de três meses antes do estudo, doença cardíaca descompensada, processo infeccioso, que tinham participado ou estivessem participando de qualquer estudo envolvendo atividade física há menos de seis meses.

O estudo foi aprovado pelo Comitê de Ética em Pesquisa da Universidade Federal do Ceará, parecer 1.113.278. CAEE: 43697715.0 .0000 .5054$.
A avaliação consistiu na coleta dos dados pessoais (idade, gênero, altura, peso, índice de massa corpórea (IMC), tempo de hemodiálise, se diabético/hipertenso ou não, uso ou não de tabaco, causas da DRC, comorbidades e medicamentos) através de uma ficha de avaliação fisioterapêutica própria. A estatura (metros) e o peso (quilogramas) foram mensurados através de um estadiômetro (600-2,100-mm model, acurácia- $1.5 \mathrm{~mm}$ ) e uma balança eletrônica modelo MRP200P (acurácia $0.1 \mathrm{~kg}$ ), respectivamente. Os participantes durante as mensurações acima, estavam usando roupa leve e sem sapatos.

\section{Em decorrência}

dessas e de outras

alterações, esses

pacientes têm

redução da força $e$

função muscular

respiratória e

periférica, bem

como, redução no

condicionamento

cardiorrespiratório.
As avaliaçóes foram realizadas por profissionais previamente treinados. É importante salientar que as mesmas foram realizadas antes do paciente entrar na sessão $\mathrm{HD}$, no segundo dia de hemodiálise da referida semana, para controle do peso, pois ao primeiro dia da semana, devido ao fim de semana, o participante teria uma maior alteração dessa variável.

Os dados respiratórios avaliados foram a condição pulmonar: medidas das pressões inspiratória e expiratória máximas (PImáx e PEmáx) e medida dos volumes e capacidades pulmonares (Capacidade Vital Forçada- CVF; Volume Expiratório Forçado no primeiro segundo- VEF1). A mensuração das pressões respiratórias máximas (PImáx e PEmáx) foi realizada através do manovacuômetro $\mathrm{MR}^{\circ} \mathrm{e}$ a avaliação dos volumes e capacidades foi feita utilizando-se o espirômetro portátil da marca ONE FLOW RANGE (Clement Clarke International) e seguiu as normas das Diretrizes para Testes de Função Pulmonar, descritas pela Sociedade Brasileira de Pneumologia e Tisiologia. Para realização do teste, o indivíduo permaneceu sentado, cabeça em posição neutra, sem flexão da cervical, utilizando um clipe nasal para evitar o vazamento de ar.

Os dados foram tabulados no programa Microsoft Excel 2010 e posteriormente analisados no programa estatístico Statistical Package for the Social Sciences, versão 17.0 (SPSS 17.0). Os resultados das variáveis estudadas foram expressos como média \pm desvio-padrão para variáveis contínuas distribuídas e frequência e porcentagem para variáveis categóricas. Para o cálculo dos valores previstos para PImáx, PEmáx, CVF e VEF1 foram utilizadas as tabelas referentes a população brasileira baseadas no estudo de Pereira (2002) ${ }^{(8)}$. Para a comparação das médias obtidas para cada variável dos diferentes gêneros foi utilizado o teste $T$ independente. Foram considerados estatisticamente significantes valores de $\mathrm{p} \leq 0,05$.

\section{RESULTADOS}

Foram incluídos 41 pacientes com DRC 
Figura 1- Fluxograma do delineamento da amostra do estudo.

\section{4 pacientes elegíveis}

28 não aceitaram participar da avaliação

\section{Participantes $(n=56)$}

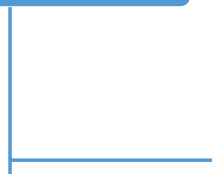

\section{Excluídos antes da data marcada para avaliação \\ 10-Desistência \\ 1-Transplante Renal \\ 3- Hospitalizado por problemas cardíacos \\ 1- Complicação infecciosa (Pneumonia)}

\section{Amostra Final $(n=41)$}

Tabela 1 - Dados demográficos gerais da amostra do estudo (Fortaleza-CE).

\section{VARIÁVEIS}

Idade (anos)

Gênero Masc, n (\%)

Peso (Kg)

$\mathrm{IMC}^{*}$

Tempo de HD (meses)

Diabéticos, n (\%)

Hipertensos, n (\%)

Uso de tabaco

Já fumou n (\%)

Nunca fumou $n(\%)$

\section{Causas da DRC}

Diabetes, $n(\%)$

Hipertensão, n (\%)

Glomerulonefrites, n (\%)

Causa não especificada, n (\%)

\section{Comorbidades}

Diabetes, n (\%)

Hipertensão, n (\%)

Doença Cardíaca, n (\%)

Doença Vascular Periférica, n (\%)

\section{Medicamentos Anti-hipertensivos:}

Inibidor de ECA**, $\mathrm{n}(\%)$

Bloqueadores de canais de cálcio, n (\%)

Beta- Bloqueadores, n (\%)
$14(34,1)$

\section{MÉDIA \pm DESVIO-PADRÃO}

$49,98 \pm 13,40$

$24(58,5)$

$74,39 \pm 17,68$

$28,18 \pm 6,16$

$28,10 \pm 20,80$

$14(34,1)$

41(100)

$16(39,0)$

25(61,0)

$14(34,1)$

$06(14,6)$

09(21,9)

$11(26,8)$

$14(34,1)$

41(100)

$11(26,8)$

$11(26,8)$

$11(26,8)$

$10(24,3)$ em hemodiálise de uma população de 84 particpantes elegíveis, porém, 28 não aceitaram participar do estudo e 15 foram excluídos previamente a data marcada par a avaliação (10 desistência; 01 transplante renal; 03 hospitalizados por problemas cardiácos e 01 por complicação infecciosa- pneumonia) (Figura 1). Os participantes apresentaram média de idade de 49,98 $\pm 13,40$ anos; de peso de 74,39 $\pm 17,68 \mathrm{~kg}$; de IMC de $28,18 \pm 6,16 \mathrm{Kg} / \mathrm{m} 2$ e de tempo de HD de 28,10 $\pm 20,80$ meses.

Dentre os pacientes avaliados, 24 $(58,5 \%)$ eram do gênero masculino, 14 $(34,1 \%)$ diabéticos e $16(39,0 \%)$ tabagista ou fizeram uso. As causas da DRC mais prevalentes foram: diabetes com 14 pacientes $(34,1 \%)$, hipertensão arterial sistêmica (HAS) em 06 (14,6\%) e as glomerulonefrites (GNF) em 09 (21,6\%). A comorbidade mais prevalente foi a HAS presente na totalidade dos pacientes e o medicamento anti-hipertensivo mais utilizado foi o inibidor da enzima conversora de angiotensina (ECA) por 14 (34,1\%). Os parâmetros de força e função pulmonares onservados foram PImáx de 79,90 $\pm 24,39 \mathrm{~cm} / \mathrm{H}_{2} \mathrm{O}, \mathrm{PE}$ máx de $71,59 \pm 23,58 \mathrm{~cm} / \mathrm{H}_{2} \mathrm{O}, \stackrel{2}{\mathrm{C} V F}$ de $2,88 \pm 1,00 \mathrm{l} / \mathrm{min}$ e VEF1 de 2,16 $\pm 0,79$ $1 /$ min (Tabela 1 ).

Aos serem feitas as análises da força muscular respiratória, separadas por gênero, foram encontrados os seguintes valores: a média da PImáx de mulheres foi de 72,35 $\pm 25,37 \mathrm{~cm} / \mathrm{H}_{2} \mathrm{O}$. Sendo o valor previsto de $84 \mathrm{~cm} / \mathrm{H}_{2} \mathrm{O}$, constatou-se que foram alcançados $85 \%$ desse valor. Quanto aos participantes do gênero masculino a média de PImáx foi de $85,25 \pm 22,68 \mathrm{~cm} / \mathrm{H}_{2} \mathrm{O}$, sendo o valor previsto de $120 \mathrm{~cm} / \mathrm{H}_{2} \mathrm{O}$, percebeu-se que foi alcançado $70 \%$ desse valor.

Em relação à PEmáx, o gênero feminino obteve média de $55,18 \pm 13,71 \mathrm{~cm} / \mathrm{H}_{2} \mathrm{O}$. $\mathrm{O}$ valor previsto foi de $115 \mathrm{~cm} / \mathrm{H}_{2} \mathrm{O}$, sendo alcançados $48 \%$ desse valor; e a média da PEmáx do gênero masculino foi de 83,21 $\pm 22,27 \mathrm{~cm} / \mathrm{H}_{2} \mathrm{O}$ com previsto de $126 \mathrm{~cm} /$ $\mathrm{H}_{2} \mathrm{O}$, obtendo-se $65 \%$ desse valor.

Quando comparadas ambas as variáveis em relação ao percentual do previsto alcançado para cada gênero, verificou-se que não há diferença significativa entre 


\section{artigo}

Campos, N.G.; Leite, L.C.; Costa, R.F.; Rebouças, E.R.N.; Miranda, L.R.; Arruda, F.L.; Ribeiro da Silva, P.R.;

Avaliação da força muscular respiratória e função pulmonar de pacientes com doença renal crônica submetidos à hemodiálise

$\begin{array}{cc}\alpha_{2} \text { Agonista Adrenérgico, } \mathrm{n}(\%) & 13(31,7) \\ \text { Vasodilatadores, } \mathrm{n}(\%) & 10(24,3) \\ \text { Parâmetros pulmonares } & \\ \text { Pimáx }\left(\mathrm{cm} / \mathrm{H}_{2} \mathrm{O}\right) & 79,90 \pm 24,39 \\ \text { Pemáx }\left(\mathrm{cm} / \mathrm{H}_{2} \mathrm{O}\right) & 71,59 \pm 23,58 \\ \text { CVF }(\mathrm{l} / \mathrm{min}) & 2,88 \pm 1,00 \\ \text { VEF1 }(\mathrm{l} / \mathrm{min}) & 2,16 \pm 0,79 \\ \text { Hemoglobina }(\mathrm{g} / \mathrm{dL}) & 10.6 \pm 1.8 \\ \text { Paratormônio Sérico }(\mathrm{pg} / \mathrm{mL}) & 275 \pm 141 \\ \text { Cálcio Sérico }(\mathrm{mg} / \mathrm{dL}) & 8.7 \pm 1.0 \\ \text { Fósforo Sérico }(\mathrm{mg} / \mathrm{dL}) & 5.7 \pm 3.1\end{array}$

IMC-Índice de massa corporal, ECA-Enzima conversora de angiotensina, DRC- doença renal crônica, PImax-pressão inspiratória máxima, PEmax- pressão expiratória máxima, CVF- capacidade vital forçada, HD- hemodiálise. Valores expressos em média đdesvio padrão.

Tabela 2 - Valores obtidos de Pimáx e Pemáx ao separar os participantes por gênero, considerando o previsto e o percentual do previsto alcançado.

\section{FORÇA MUSCULAR RESPIRATÓRIA}

\section{VARIÁVEIS}

$\begin{array}{cr}\text { Pimáx-cm/ } \mathrm{H}_{2} \mathrm{O}(\mathrm{M}) & 72,35 \pm 25,37 \\ \text { Pimáx cm/ } / \mathrm{H}_{2} \mathrm{O}(\mathrm{H}) & 85,25 \pm 22,68 \\ \text { Pemáx- } \mathrm{cm} / \mathrm{H}_{2} \mathrm{O}(\mathrm{M}) & 55,18 \pm 13,71 \\ \text { Pemáx- } \mathrm{cm} / \mathrm{H}_{2} \mathrm{O}(\mathrm{H}) & 83,21 \pm 22,27\end{array}$

PREVISTO (CM/
$\left.\mathrm{H}_{2} \mathrm{O}\right)$

84

120

115

126

\section{DO PREVISTO ALCANÇADO}

$85 \%$

$70 \%$

$48 \%$

$65 \%$

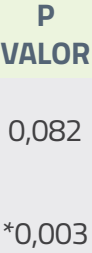

${ }^{*} 0,003$

$(M)$ = Mulheres; $(H)=$ Homens. Plmax-pressão inspiratória máxima, Pemax- pressão expiratória máxima. Valores expressos em média \pm desvio padrão. $(*)$ Há diferença significativa entre homens e mulheres quando comparados em relação aos valores dos previstos alcançados para a variável Pemáx.. Teste T independente. Foram considerados estatisticamente significantes valores de $p \leq 0,05$.

\section{Tabela 3 - Valores obtidos de CVF e VEF1 ao separar os participantes por} gênero, considerando o previsto e o percentual do previsto alcançado.

\section{VARIÁVEIS}

\begin{tabular}{ccccc} 
CVF -L/min (M) & $2,22 \pm 0,65$ & 3,19 & $69 \%$ & \multirow{2}{*}{0,034} \\
CVF- L/min (H) & $3,35 \pm 0,95$ & 3,57 & $93 \%$ & \\
VEF1 -L/min (M) & $1,63 \pm 0,57$ & 2,47 & $65 \%$ & ${ }^{*} 0,024$ \\
VEF1-L/min (M) & $2,53 \pm 0,71$ & 2,87 & $95 \%$ &
\end{tabular}

$(M)=$ Mulheres; $(H)=$ Homens. CVF- capacidade vital forçada, HD- hemodiálise. Valores expressos em média đdesvio padrão. ${ }^{*}$ ) Há diferença significativa entre homens e mulheres quando comparados em relação aos valores dos previstos alcançados para ambas as variáveis. Teste $T$ independente. Foram considerados estatisticamente significantes valores de $p \leq 0,05$.

homens e mulheres em relação à PImáx, porém foi significativa em relação a PEmáx $(\mathrm{p}=0,082, \mathrm{p}=0,003$, respectivamente) (Tabela 2).

Em relação às análises da função pulmonar separadas por gênero foi verificada: a média da CVF das mulheres de 2,22 \pm $0,65 \mathrm{l} / \mathrm{min}$. O previsto foi de $3,19 \mathrm{l} / \mathrm{min}$, sendo alcançados $69 \%$ desse valor. Quanto aos homens a CVF foi de 3,35 $\pm 0,95 \mathrm{l} /$ min tendo como previsto um valor de 3,57 $1 / \mathrm{min}$, portanto foram obtidos $93 \%$ do previsto. Ao verificar os valores de VEF1; nas mulheres a média foi de 1,63 $\pm 0,57 \mathrm{l} /$ min e o previsto de $2,471 / \mathrm{min}$; foi evidenciado, portanto que foram contemplados $65 \%$ desse valor. A média do VEF1 dos homens foi de 2,53 $\pm 0,71 \mathrm{l} / \mathrm{min}$, sendo o previsto de $2,87 \mathrm{l} / \mathrm{min}$, o que revela que foram atingidos $95 \%$ desse valor.

Quando esses valores foram comparados em relação ao percentual do previsto alcançado para cada gênero, foi constatado que há diferença significativa entre homens e mulheres, para ambas as variáveis CVF e VEF1 ( $p=0,034$ e $p=0,024$, respectivamente), com $\mathrm{p} \leq 0,05$ (tabela 3 ).

\section{DISCUSSÃO}

Vários estudos documentaram que pacientes com DRC submetidos a HD apresentam redução da força muscular respiratória e periférica, baixo condicionamento cardiorrespiratório e redução na capacidade funcional que influenciam direta e negativamente no desempenho de suas atividades de vida diária, bem como, na qualidade de vida $(6,9,10)$.

O comprometimento da função pulmonar pode resultar diretamente de toxinas urêmicas circulantes ou pode resultar indiretamente da sobrecarga de fluidos, anemia, supressão imune, calcificação extra óssea, desnutrição, distúrbios eletrolíticos e/ou desequilíbrios ácidos-básicos, que são problemas comuns em pacientes com DRC hemodialíticos ${ }^{(2)}$.

O sistema respiratório é afetado tanto pela doença como pelo tratamento hemodialítico, sendo assim, a força muscular respiratória e a endurance apresentam-se diminuídas nos pacientes com DRC quando comparados com indivíduos saudáveis (12) .No presente estudo, ao se comparar os valores obtidos de PImáx e PEmáx, com participantes separados por gênero e considerando o previsto e o percentual do previsto alcançado, foi constatado que a PEmáx é mais comprometida do que a PImáx, corroborando com o estudo de Cunha et al. (2009) (13) ,cujos pacientes com DRC em HD apresentaram importantes alterações quanto à força muscular respiratória, principalmente na força expiratória, tendo apresentado níveis muito abaixo dos preditos ( $42,8 \%$ do previsto) o que pode estar associado à diminuição da força da musculatura esquelética, característica da miopatia urêmica. 
Kovelis et al. (2008) (12) realizou a avaliação da função pulmonar e da força muscular respiratória de 17 pacientes com DRC hemodialíticos com o intuito de correlacioná-las com a variação de peso ligada à realização de HD e também verificar a correlação entre o tempo de hemodiálise e possíveis alterações respiratórias. Como um dos resultados principais, o estudo demonstrou correlação entre a perda de força muscular respiratória e o tempo de tratamento por hemodiálise, isto é, o maior tempo de hemodiálise está associado à diminuição da força muscular respiratória. No presente estudo observou-se que os pacientes possuem um pouco mais de dois anos de tratamento hemodialítico, e que os homens possuem $70 \%$ do previsto para força muscular inspiratória, o que configura segundo Sousa (2002) (14), fraqueza muscular leve. Especula-se, portanto, a possibilidade da diminuição da força muscular com a evolução da doença e o aumento do período de tratamento.

Diante do supracitado, percebe-se que a uremia e a diálise interferem no estímulo respiratório e na mecânica, função muscular e troca de gases. A HD de alguma forma, em algum momento, afetará o sistema respiratório dos pacientes submetidos a esse tratamento ${ }^{(15)}$. As alterações pulmonares mais encontradas são a limitação ao fluxo aéreo, desordens restritivas, redução da capacidade de difusão pulmonar, diminuição da endurance e força muscular respiratória ${ }^{(12,16,17)}$.

Uma pesquisa realizada por Morais et al $(2011)^{(18)}$ avaliou a força muscular respiratória em pacientes diabéticos tipo $2 \mathrm{e}$ foi verificada uma prevalência de $31 \%$ de fraqueza muscular inspiratória nesses indivíduos. Na presente pesquisa, foi observado que a DM e HAS foram as principais causas da DRC e esse resultado concorda com o estudo de Posser et al., $2016^{(9)}$ que constatou similaridade nas causas da doença. Sugestiona-se, nesse caso, que a DM possa ser um fator influenciador para a diminuição da força muscular respiratória no presente estudo.

Vale salientar que ao serem comparados mulheres e homens em relação à força muscular respiratória as mulheres apresentam uma redução significativa mais acentuada da Pemáx. Essas diferenças nos valores da pressão expiratória máxima entre os gêneros podem estar relacionadas e também exacerbadas, além da condição clínica, pela desigualdade na quantidade de massa magra, já que, em geral, os homens têm maior quantidade de massa muscular e, consequentemente, mais força que as mulheres quando se exige uma força expiratória forçada ${ }^{(19)}$.

Os resultados encontrados na presente pesquisa apontam o que alguns autores já descreviam ao verificar a presença de redução da força muscular respiratória e mudanças negativas na função pulmonar em pacientes hemodialíticos ${ }^{(20,21)}$. A consequência desse comprometimento na musculatura respiratória junto a outros problemas pulmonares e sistêmicos contribui para a diminuição da capacidade pulmonar, devido ao prejuízo na função desse sistema ${ }^{(22,23)}$.

Tal condição pode ser explicada devido à miopatia urêmica, presente nos pacientes com DRC submetidos a HD e caracterizada por uma fraqueza generalizada que aparece como fator importante para a redução da força dos músculos respiratórios. Devido a essa condição, as reduções e limitações do fluxo aéreo, principalmente do VEF1, encontradas nas mulheres do presente estudo podem estar associadas ao atraso da contração da fibra muscular por conta da fraqueza desses músculos, especialmente os expiratórios que tiverem um valor de força bem abaixo do previsto ${ }^{(24,25)}$.

Os primeiros relatos sobre função respiratória no paciente dialítico, no Brasil, começaram no final da última década e tinham, em sua maioria, o objetivo de estudar a função pulmonar associada ao tempo de HD ${ }^{(14,29,24)}$. Em um dos estudos, Cury et al. (2010) ${ }^{(19)}$ avaliaram a função pulmonar nesses pacientes e foi possível afirmar que ocorreu uma diminuição na capacidade vital forçada (CVF), no volume expirado forçado no primeiro segundo (VEF1) e na ventilação voluntária máxima (VVM), apontada através da espirometria.

Ao se tratar da CVF e do VEF1 nos pacientes hemodialíticos, autores propõem que os principais distúrbios que podem estar associados a baixos resultados dessas duas variáveis nesse perfil de pacientes, no caso das mulheres da presente pesquisa, podem ser explicados pelas condições subclínicas como: diminuição da albumina sérica com consequente desequilíbrio hídrico e protéico na microcirculação, fibrose intersticial e calcificações do parênquima pulmonar e árvore brônquica e ou fibrose por corticoterapia, caso paciente faça uso deste ${ }^{(21)}$.

Outro fator que merece destaque é que além do comprometimento muscular respiratório que pode explicar a diminuição da função pulmonar, existe também o acúmulo de líquido interdialítico que leva ao aumento da pressão hidrostática e altera a permeabilidade capilar pulmonar, prejudicando os volumes e capacidades pulmonares ${ }^{(26)}$.

Em contrapartida, os participantes do gênero masculino, em relação à função pulmonar mostraram valores de CVF e VEF1 dentro dos padróes de normalidade e melhores do que as mulheres. Sugestiona-se que esses valores possam ser explicados pela melhor força muscular expiratória encontrada nos homens.

O número reduzido de participantes que foram avaliados, devido à necessidade de serem voluntários sem qualquer doença pulmonar e ou cardíaca prévia e que tivessem condições cognitivas e físicas de realizar os dois testes utilizados, foi um fator limitante para esse estudo. Porém, como ponto relevante o estudo fomenta a implantação de rotina da fisioterapia na assistência ao paciente com doença renal crônica submetido à hemodiálise, pois já é sabido sobre o prejuízo na força muscular respiratória e função pulmonar sofrida por esse grupo de pacientes.

\section{CONCLUSÃO}

Quanto à força muscular respiratória, os homens com DRC hemodialítica apresentaram força muscular inspiratória reduzida em relação ao previsto, caracterizando fraqueza muscular leve. Quando comparadas ambas as variáveis (PImáx e PEmáx) em relação ao percentual do previsto alcançado, para cada gênero, verifi- 


\section{artigo}

Campos, N.G.; Leite, L.C.; Costa, R.F.; Rebouças, E.R.N.; Miranda, L.R.; Arruda, F.L.; Ribeiro da Silva, P.R.;

Avaliação da força muscular respiratória e função pulmonar de pacientes com doença renal crônica submetidos à hemodiálise

cou-se que não há diferença significante entre homens e mulheres em relação a PImáx, porém há uma diferença em relação a PEmáx, obtendo os homens um melhor resultado nessa última variável, embora ainda fora dos padrões de normalidade para ambos os gêneros.

Quando analisada a função pulmonar, constatou-se que o gênero masculino possui função pulmonar relacionado aos valores de CVF e VEF1 dentro da normalidade enquanto o gênero feminino apre- senta redução da função pulmonar. Ao serem comparados os diferentes gêneros em relação ao percentual do previsto, foi constatado que há diferença significativa entre homens e mulheres, para ambas as variáveis acima citadas.

\section{REFERÊNCIAS}

1. Of, O. J. O. S.; Kidney Disease: Improving Global Outcomes (KDIGO) CKD Work Group. KDIGO 2012 Clinical Practice Guideline for the Evaluation and Management of Chronic Kidney Disease. Kidney International Supplements.3(1), (2013) 4-4.

2. Yilmaz S, Yildirim Y, Yilmaz Z, Kara AV, Taylan M, Demir M. Pulmonary Function in Patients with End-Stage Renal Disease: Effects of Hemodialysis and Fluid Overload. Medical Science Monitor: International Medical Journal of Experimental and Clinical Research, Diyarbakir. 7(22), (2016)2779-2784.

3. Zhenzhen Qiu, Kai Zheng, Haoxiang Zhang, Ji Feng, Lizhi Wang, and Hao Zhou, "Physical Exercise and Patients with Chronic Renal Failure: A Meta-Analysis," BioMed Research International.2017, Article ID 7191826, 8 pages.

4. Gesualdo, G. D. et al. Factors Associated With the Quality of Life of Patients Undergoing Hemodialysis. Texto \& Contexto Enfermagem (26)2, (2017)1-10.

5. Abdalla, M. E. Evaluation of pulmonary function in renal transplant recipients and chronic renal failure patients undergoing maintenance hemodialysis. Egyptian Journal of Chest Diseases and Tuberculosis.62(1), (2013)145-150..

6. De Medeiros, A. I. C. et al. Inspiratory muscle training improves respiratory muscle strength, functional capacity and quality of life in patients with chronic kidney disease: a systematic review. Journal of Physiotherapy.63(2)76-83.

7. Pellizzaro, C. O.; Thomé, F. S.; Veronese, F. V. Effect of Peripheral and Respiratory Muscle Training on the Functional Capacity of Hemodialysis Patients. Renal Failure. 35(2),(2013).

8. Pereira, C. A. D. C. Espirometria. Jornal Brasileiro de Pneumologia.28 (2002.)n. supl 3, p. S1-S82.

9. Posser, S. R. et al. Functional capacity, pulmonary and respiratory muscle strength in individuals undergoing hemodialysis. 3(2016) 343-350.

10. Oliveira, A. P. B. et al. Quality of life in hemodialysis patients and the relationship with mortality, hospitalizations and poor treatment adherence. Jornal Brasileiro de Nefrologia. 38(4),(2016).

11. Krittayaphong, R. et al. Prevalence of chronic kidney disease associated with cardiac and vascular complications in hypertensive patients: a multicenter, nation-wide study in Thailand. BMC Nephrology.18(1),(2017)115, 2017.

12. Kovelis, D.; Pitta, F.; Probst, V. Pulmonary function and respiratory muscle strength in chronic renal failure patients on hemodialysis. Jornal Brasileiro de Pneumologia. 34 (2008) 907-912.

13. Cunha, M. S.; Andrade, V., Guedes, C. A. V.; Meneghetti, C. H. Z.; Aguiar, A. P.; Cardoso, A. L. Avaliação da capacidade funcional e da qualidade de vida em pacientes renais crônicos submetidos a tratamento hemodialítico. Fisioterapia e pesquisa. 16(2),(2009) 155-60.

14. Souza RB. Pressões Respiratórias Estáticas Máximas. J Pneumol.(2002);28(Supl 3):S155-65.

15. Lang, S. M.; Becker, A.; Fischer, R.; Huber, R. M.; Schiffl, H. Acute effects of he-modialysis on lung function in patients with end-stage renal disease. Wien Kin Wo-chenschr. Alemanha. 118(3), (2006) 108-113.

16. Jatobá, P. C.; Amaro, W. F.; De Andrade, A. A.; Cardoso, F. P. F.; Monteiro, A.M.H. Oliveira, M.M. Evaluation of pulmonary function, respiratory muscle strength and six-minute walk test in patients with chronic renal disease on hemodialysis. J Bras Nefrol. Brasília. 30(4), (2008) 280-287.

17. Schardong, J. T.; Lukrafka, L. J.; Garcia, D. V. Evaluation of pulmonary function and quality of life in patients with chronic kidney disease undergoing hemodialysis. J Bras Nefrol. 21(2008) 40-7.

18. Morais, et al. Avaliação da força muscular em idosos com DM2. Revista contexto e saúde 10 (20), (2011) 169-78.

19. Simões, R. P. et al. Influence of age and sex on respiratory muscle strength.J Bras Nefrol.14(1),(2007)36-41.

20. Cury. J.L.; Brunetto, A.F.; Aydos, R.D. Efeitos negativos da insuficiência renal crônica sobre a função pulmonar e a capacidade funcional. Revista Brasileira de Fisioterapia. São Carlos.14(2),(2010) 91-98.

21. Karacan, Ö.; Tutal, E.; Colak, T.; Sezer, S.; Eyuboglu, FÖ.; Haberal, M. Pulmonary function in renal transplant recipients and end-stage renal disease patients undergoing maintenance dialysis. Paper present data: Transplantation proceedings. 38(2),(2006) 396-400.

22. Sakkas, G. K., Sargean, A. J., Mercer, T. H., Baal, D., Koufaki, P., Karatzafer, C., et al. Changes in muscle morphology in dialysis patients after 6 months of aerobic exercise training. Nephrol Dial Transplant. 18(9),(2003)1854-61.

23. Kemp, G. J.; Crowe, A. V.; Anijeet, H. K.; Gong, Q. Y.; Bimson, W. E.; Frostick, S. P. et al. Abnormal mitochondrial function and muscle wasting, but normal contractile efficiency, in haemodialysed patients studied non-invasively in vivo. Nephrol Dial Transplant.19(6),(2004) 1520-7.

24. Rocha, C. B. J.; Araújo, S. Evaluation of maximum respiratory pressures in chronic kidney desease patients in the pre and post-hemodialysis moments.J Bras Nefrol, v. 32, p.107-13, 2010.

25. Kalender, B.; ERK, M.; Pekpak, M. A, et al. The effect of renal transplantation on pulmonar function. Nephron. 90 (2002)72-77.

26. S. Yılmaz, Y. Yildirim, Z. Yilmaz, A.V. Kara, M. Taylan, M. Demir, M. Coskunsel, A.K. Kadiroglu, M.E. Yilmaz, Pulmonary function in patients with end-stage renal disease: effects of hemodialysis and fluid overload, Med. Sci. Monit. 22 (2016)2779-278. 